\title{
High-molecular weight hyaluronan attenuates tubulointerstitial scarring in kidney injury
}

\author{
Xinyi Wang, ${ }^{1}$ Swathi Balaji, ${ }^{1}$ Emily H. Steen, ${ }^{1}$ Alexander J. Blum, ${ }^{1}$ Hui Li, ${ }^{1}$ Christina K. Chan, ${ }^{2}$ \\ Scott R. Manson, ${ }^{3}$ Thomas C. Lu, ${ }^{1}$ Meredith M. Rae, ${ }^{1}$ Paul F. Austin, ${ }^{3}$ Thomas N. Wight, ${ }^{2}$ \\ Paul L. Bollyky, Jizhong Cheng, ${ }^{5}$ and Sundeep G. Keswani' \\ 'Laboratory for Regenerative Tissue Repair, Division of Pediatric Surgery, Department of Surgery, Texas Children's \\ Hospital/Baylor College of Medicine, Houston, Texas, USA. Matrix Biology Program, Benaroya Research Institute, \\ Seattle, Washington, USA. 'Division of Pediatric Urology, Texas Children's Hospital/Baylor College of Medicine, Houston, \\ Texas, USA. ${ }^{4}$ Division of Infectious Diseases, Department of Medicine, Stanford University School of Medicine, Stanford, \\ California, USA. ${ }^{5}$ Section of Nephrology, Department of Medicine, Baylor College of Medicine, Houston, Texas, USA
}

Renal fibrosis features exaggerated inflammation, extracellular matrix (ECM) deposition, and peritubular capillary loss. We previously showed that IL-10 stimulates high-molecular weight hyaluronan (HMW-HA) expression by fibroblasts, and we hypothesize that HMW-HA attenuates renal fibrosis by reducing inflammation and ECM remodeling. We studied the effects of IL-10 overexpression on HA production and scarring in mouse models of unilateral ureteral obstruction (UUO) and ischemia/reperfusion (I/R) to investigate whether IL-10 antifibrotic effects are HA dependent. C57BL/6] mice were fed with the HA synthesis inhibitor, 4-methylumbelliferone (4MU), before UUO. We observed that in vivo injury increased intratubular spaces, ECM deposition, and $\mathrm{HA}$ expression at day 7 and onward. IL-10 overexpression reduced renal fibrosis in both models, promoted HMW-HA synthesis and stability in UUO, and regulated cell proliferation in I/R. 4-MU inhibited IL-10-driven antifibrotic effects, indicating that HMW-HA is necessary for cytokinemediated reduction of fibrosis. We also found that IL-10 induces in vitro HMW-HA production by renal fibroblasts via STAT3-dependent upregulation of HA synthase 2. We propose that IL-10induced HMW-HA synthesis plays cytoprotective and antifibrotic roles in kidney injury, thereby revealing an effective strategy to attenuate renal fibrosis in obstructive and ischemic pathologies.

Conflict of interest: The authors have declared that no conflict of interest exists.

Copyright: () 2020, American Society for Clinical Investigation.

Submitted: January 10, 2020

Accepted: May 7, 2020

Published: June 18, 2020.

Reference information: /CI Insight. 2020;5(12):e136345.

https://doi.org/10.1172/jici.

insight.136345.

\section{Introduction}

The development, progression, and complications of fibrosis account for over $45 \%$ of mortality in developed countries $(1,2)$. Renal glomerulosclerosis or tubulointerstitial fibrosis are often the result of chronic kidney disease (CKD), which has a global prevalence of approximately $10 \%$. Renal fibrosis is characterized by uncontrolled inflammation, peritubular capillary rarefaction, and functional failure caused by excessive extracellular matrix (ECM) deposition (3-6). Current transplantation and dialysis treatments do not address the underlying pathophysiology of renal fibrosis and CKD, nor do they take advantage of the kidneys' inherent capacity for postinjury cytoprotective and regenerative functions (7-9). Notably, we and others have shown that interleukin-10 (IL-10) and hyaluronan (HA) are critical for regenerative healing in most organs (10-13). This means that elucidating innate mechanisms that regulate regenerative tissue repair could provide new knowledge that would lead to adjuvant treatments to reduce fibrosis and improve CKD patient outcomes and quality of life $(14,15)$.

HA, a glycosaminoglycan composed of linearly repeating disaccharides within the ECM, has gained attention since the 1990s for its involvement in inflammation and multiorgan fibrosis (16-19). HA has pleiotropic functions that depend on its molecular mass and tissue distribution (20). For instance, highmolecular weight HA (HMW-HA) is produced by HA synthases (Has) 1 or 2 and exerts antiinflammatory and antifibrotic effects, while low-MW HA (LMW-HA; $<1,000 \mathrm{kDa}$ ) is produced mainly by Has3 and causes inflammation and fibrosis $(11,21-24)$. Moreover, HA stability is regulated by hyaluronidases $1-4$, which cleave HA into oligosaccharides $(25,26)$. Physiologically, HA is present in the interstitial renal 
medulla/papilla and to a lesser extent in the cortex and rapidly turns over when systemic hydration levels change. Many chronic inflammatory diseases like CKD are accompanied by total changes in HA MW variants (27-30). For instance, studies in rats showed that HA levels gradually increase and peak at day 11 after ureteral obstruction, then decrease to undetectable levels by day 22. However, the role that HA MW plays in kidney fibrosis remains unclear (31).

To further assess the in vitro and in vivo requirement of HA during fibrotic processes, cell cultures and mice were treated with 4-methylumbelliferone (4-MU). An effective Has inhibitor, 4-MU is known to systemically reduce HA levels by depleting cellular uridine diphosphate-glucoronic (UDP-glucuronic) acid levels $(20,32)$ and inhibiting the transcription of Has, UDP-glucose pyrophosphorylase, and dehydrogenase (33-37).

Fibroblasts and myofibroblasts are the primary mediators of fibrosis, accounting for the majority of ECM production in the kidney, and are likely candidates for the production of HA in response to injury $(14,38)$. Myofibroblasts in particular contribute to renal fibrosis by recruiting inflammatory macrophages and remodeling the ECM through collagen, proteoglycan/glycosaminoglycan, and matrix metalloproteinase production (38-41). However, when fibroblasts are treated with IL-10, high concentrations of HMW-HA are produced and tissue regeneration ensues (11). Although other studies have reported that IL-10 can attenuate renal fibrosis, the contribution of HMW-HA to the process has been largely unexplored $(42,43)$. We hypothesize that sustained HMW-HA levels promote antifibrotic tissue remodeling in response to renal injury.

To test this hypothesis, we examined the role of HMW-HA using unilateral ureteral obstruction (UUO) and ischemia/reperfusion (I/R) as bona fide renal fibrosis models of murine chronic and acute kidney injury, respectively. We ultimately used IL-10-mediated HA overexpression (gain-of-function) and 4-MUdriven Has inhibition (loss-of-function) experiments to determine whether HMW-HA is necessary and sufficient to influence the direction of postinjury tissue repair.

\section{Results}

UUO injury increases HA deposition by promoting HMW-HA synthesis. We performed UUO on C57BL/6J mice and harvested the kidneys to assess HA synthesis and degradation in the renal parenchyma. Figure $1 \mathrm{~A}$ shows images of control (uninjured) and post-UUO kidney tissue sections (days 3, 7, and 14) stained with periodic acid-Schiff (PAS), Masson's trichrome (trichrome), or HA binding protein (HABP), which were subsequently quantified (Figure 1B). PAS staining revealed that in comparison with controls, day 14 UUO kidneys showed higher interstitial thickening, decreased cellularity, and 1.74-fold larger intratubular spaces, indicating intratubular fibrosis $(P<0.01)(44)$. Trichrome staining of UUO kidneys from day 7 onward revealed greater ECM deposition than uninjured controls, peaking at day 14 post-UUO. Next, we performed immunohistochemistry (IHC) using HABP to detect HA levels (45-47), which showed higher total HA accumulation in the cortex of UUO kidneys than uninjured controls at day 7 (Figure 1). To examine whether HA levels were increased, total HA was extracted from injured kidneys at each time point and quantified by an ELISA-like test. We found that in comparison with uninjured controls, HA concentration was significantly increased at day 3 post-UUO injury (Figure 1C) and peaked at day 14 (controls vs. day 3 [3D] UUO vs. 7D UUO vs. $14 \mathrm{D}$ UUO in $\mathrm{ng} / \mathrm{mL} / \mathrm{mg}, 20.46 \pm 6.49$ vs. $216.79 \pm 76.82$ vs. $260.70 \pm 44.24$ vs. $341.31 \pm 50.09$, respectively).

To assess the MW of the increased HA after UUO, we performed 2 independent analyses. First, we examined the extracted HA from day 3 and 7 UUO kidneys by size-exclusion chromatography and then quantified the column fractions by an ELISA-like HA assay (Figure 1D). Our data showed that control samples contained mostly LMW-HA variants with a peak at $250 \mathrm{kDa}\left(\mathrm{K}_{\mathrm{av}} 0.7\right)$, while UUO extracts contained HMW-HA variants that were at least $1500 \mathrm{kDa}\left(\mathrm{K}_{\mathrm{av}}\right.$ 0.15). Moreover, we observed that the HA concentration in our UUO model at day 3 was higher than at day 7. Our observed LMW- versus HMW-HA dichotomy was confirmed by gel electrophoresis, which revealed a light band of LMW-HA between 150 and $500 \mathrm{kDa}$ in control kidney extracts (Figure 1E, lane 3) in contrast to post-UUO kidney extracts, which exhibited a higher MW range and band intensity among all UUO samples tested. Consistently, extracted HA from UUO samples revealed significantly higher MW-HA variants (lanes 4-6) than those of uninjured controls, while day 3 post-UUO confirmed the highest MW-HA levels in quantitative ELISA-like analysis (Figure 1D).

We then investigated how changes in HA MW reflect Has1-3 and hyaluronidase 1-2 mRNA expression (Figure 1F) and found that in comparison with controls, normalized post-UUO Has 1-3 expression 
A

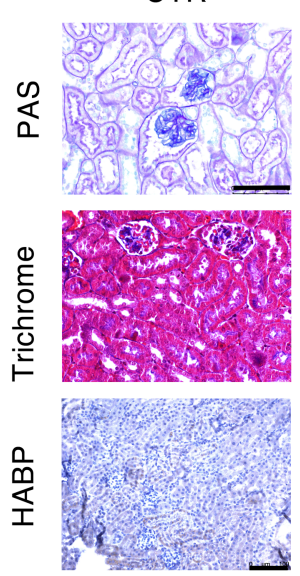

C
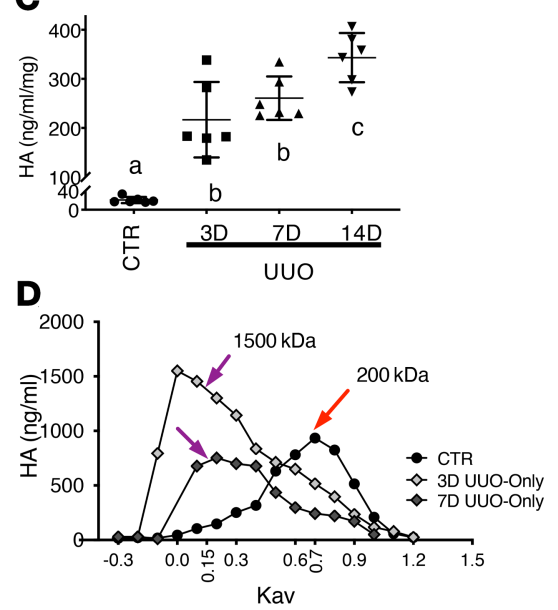

7D UUO
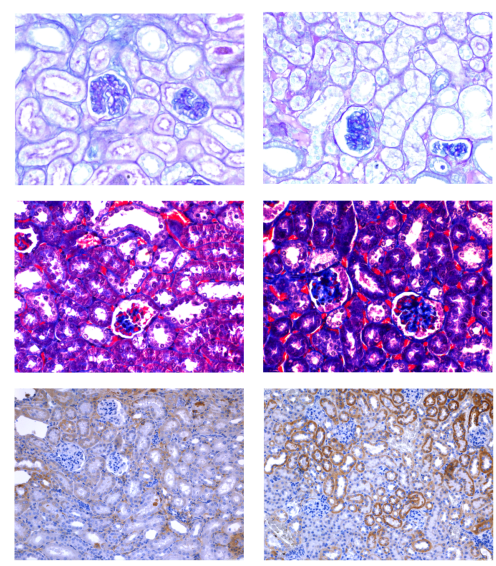

$\mathbf{E}$
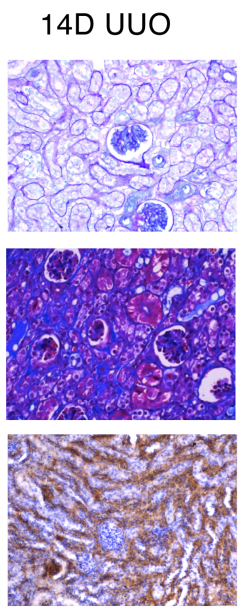

B Intratubular Space
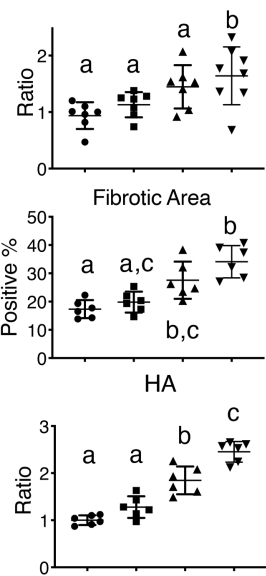

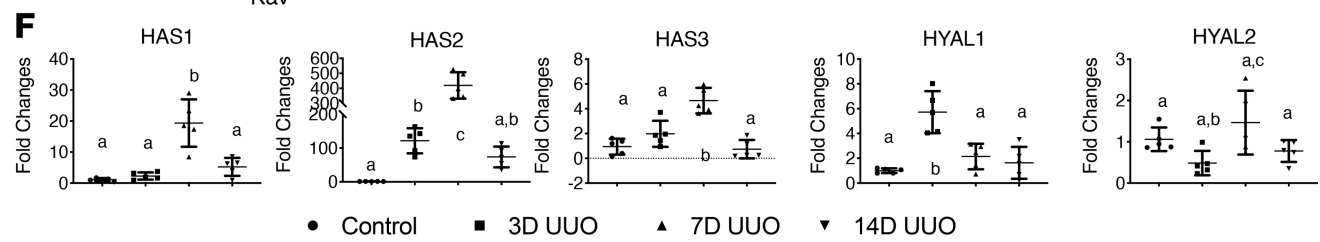

Figure 1. Increased HA in UUO-induced renal fibrosis. (A) Images of PAS, trichrome, and HABP staining of the cortex from control and UUO kidneys show fibrotic remodeling and HA accumulation after UUO injury. Scale bars: $75 \mu \mathrm{m}$ for PAS and trichrome staining, $100 \mu \mathrm{m}$ for HABP to depict broader HA changes during the process. (B) Quantitative assessment of PAS intratubular space ( $0.94 \pm 0.23$ vs. $1.13 \pm 0.22$ vs. $1.48 \pm 0.38$ vs. $1.64 \pm 0.51)$, trichrome fibrotic area $(17.33 \% \pm 3.18 \%$ vs. $19.82 \% \pm 3.71 \%$ vs. $27.56 \% \pm 6.57 \%$ vs. $34.11 \% \pm 5.72 \%)$, and HABP-detected HA levels ( $1.01 \pm 0.09$ vs. $1.28 \pm 0.23$ vs. $1.85 \pm 0.29$ vs. $2.45 \pm 0.22) . P<0.0001$. (C) Extracted HA concentration (ng/mL/mg) from control and 3-, 7-, and 14-day UUO kidneys, measured by a modified ELISA HA test kit. (D) Plots of total incorporated $\left[{ }^{3} \mathrm{H}\right]$ in labeled HA samples were used to determine the relative MW of HA synthesized by control and 3D UUO kidneys ( $n \geq 3$ per condition) using Sephacryl S-1000 chromatography. Data were plotted as $\mathrm{HA}$ concentration versus the partition coefficient $\left(\mathrm{K}_{\mathrm{ay}}\right)$, showing an increase in $\mathrm{HA}$ size distribution in samples with UUO injury. (E) The extracted HA from control and 3-, 7-, and 14-day untreated and treated UUO kidneys is shown on a $0.5 \%$ agarose gel electrophoresis, supporting our chromatography results. (F) Relative mRNA level of Has1-3 and hyaluronidases (Hyal1-2) in untreated control and UUO kidneys; $n \geq 3$ per sex per condition; $P<$ 0.01 . All data were analyzed by 1-way ANOVA with post hoc Tukey's test for differences between groups. Groups annotated with different letters indicate that they are significantly different.

was upregulated, and Has2 specifically increased by 100-fold at day 7. Of note, Has 1 and 2 expression were reduced at day 14 post-UUO but remained higher than in controls; hyaluronidase 1 expression peaked at day 3 , and hyaluronidase 2 did not change by more than 2 -fold throughout the study. Collectively, our data suggest that UUO triggers HMW-HA production via Has1 and 2 and that HA synthesis likely dominates over degradation. 
IL-10-driven HMW-HA attenuates renal fibrosis in UUO mice. We observed that synthesis of HMW-HA was induced in the early time points after UUO, but when HMW-HA production began to decrease at later time points, fibrosis increased. To determine whether increasing and sustaining HMW-HA production can attenuate fibrosis in UUO, we overexpressed IL-10 in vivo to promote HMW-HA synthesis. Images of day 14 uninjured controls, UUO-only, and IL-10-treated UUO kidneys stained with PAS, trichrome, $\alpha$-smooth muscle actin ( $\alpha$-SMA), and HABP showed that in contrast to UUO-only kidneys, IL-10-treated UUO kidneys exhibited less intratubular space and lower ECM deposition at day 14 (Figure 2A). We also found that IL-10-treated UUO kidneys had significantly lower $\alpha$-SMA expression than UUO-only kidneys. Further, IL-10-treated UUO kidneys exhibited the most pronounced HA staining (Figure 2A), which could be due to both UUO injury and IL-10-driven HMW-HA production. Indeed, quantitative assessment of intratubular spaces, fibrotic area, $\alpha$-SMA staining, and HA levels supported our findings (Figure 2B). Moreover, IL-10 treatment led to higher Has 1 and 2 mRNA expression (Figure 2C) at day 14 than in UUO-only samples, strongly suggesting that IL-10 induces and sustains HMW-HA production via Has1 and 2 activation. In keeping with these findings, all UUO kidneys expressed lower Has3 and hyaluronidase 1 mRNA levels than controls with or without IL-10, while hyaluronidase 2 was not significantly changed. Our collective data indicate that IL-10 upregulates Has 1 and 2 expression in vivo and promotes longer term HMW-HA production, which could play a cytoprotective role in renal injury.

Next, we examined HA levels by an ELISA-like test in UUO and IL-10-treated UUO kidneys (Figure 2D). HA levels for both groups increased and were sustained over time until day 7, and the amount of HA in IL-10-treated UUO kidneys continued to rise after day 14. More strikingly, IL-10-treated kidneys showed a $60 \%$ HA increase at day 21 post-UUO compared with UUO-only controls. These results further support our hypothesis that IL-10 treatment of UUO kidneys promotes high and sustained HMW-HA production, which contributes to reduced fibrosis $(48,49)$.

Gain of function by lenti-IL-10 overexpression in kidneys is heterogeneous but sufficient. To test whether a single dose of lenti-GFP-IL-10 resulted in consistent in vivo IL-10 overexpression in kidney tissues, cohorts of lenti-GFP (control vector) and lenti-GFP-IL-10-transduced mouse kidneys were tested for gene expression at day 3 post-UUO. We observed strong GFP expression in the cortex with single-dose lenti-GFP-IL-10injected kidney tissues at day 3 post-UUO (Figure 3A), as compared with lenti-GFP-treated UUO tissues. Image data were quantified (Figure $3 \mathrm{~B}$ ) and revealed that the strong GFP-IL-10 signal was 70-fold higher than the lenti-GFP kidney sections. Next, we quantified IL-10 expression in the kidney cortex by ELISA and showed that in comparison with lenti-GFP kidneys, IL-10 expression in the lenti-GFP-IL-10-transduced kidney cortex was significantly increased at day 3 post-UUO (Figure 3C, lenti-GFP vs. lenti-GFP-IL-10 in $\mathrm{pg} / \mathrm{mg}$ of tissue weight, $1.27 \pm 0.54$ vs. $4.16 \pm 1.75)$.

In vivo inhibition of $H A$ synthesis reveals that $I L-10$ antifibrotic effects are $H A$ dependent. To investigate whether the regenerative effects of IL-10 after UUO injury depend on HA, loss-of-function experiments were performed by inhibiting endogenous HA synthesis with 4-MU. UUO was performed after 6 weeks of feeding mice with a diet containing 4-MU, which continued until day 14 post-UUO $(33,37)$. IHC analyses (Figure 4A) showed that HA levels were lower in 4-MU-fed UUO mice than control diet-fed UUO mice, while trichrome and $\alpha$-SMA staining of 4-MU-fed UUO mice revealed higher interstitial ECM deposition and $\alpha$-SMA levels. Strikingly, in contrast to mice fed with control diet, IL-10 treatment failed to attenuate interstitial ECM deposition or fibrosis in UUO-injured mice fed with 4-MU, strongly suggesting that the antifibrotic effects of IL-10 are HA dependent. Next, to determine whether the canonical antiinflammatory function of IL-10 was similarly affected by 4-MUmediated inhibition of HA synthesis, we assessed the expression of F4/80, a mouse macrophage proinflammatory marker (Figure 4B). We found that in comparison with uninjured controls, UUO increased $\mathrm{F} 4 / 80^{+}$macrophage invasion but that invasion was effectively reduced by IL-10 treatment in the 4-MU-fed mice kidney tissue, even though we did not observe differences in fibrotic outcomes shown previously (Figure 2B vs. Figure 4C). Our collective data indicate that HA synthesis is necessary and sufficient to transduce IL-10 antifibrotic signaling effects in response to renal injury; however, IL-10's antiinflammatory functions operate independently from the effect on HA.

We then compared HA synthases and hyaluronidase expression between UUO-only and IL-10-treated UUO kidneys in 4-MU-fed mice and found that only Has1 was significantly upregulated with IL-10 treatment (Figure 4D). However, Has1 upregulation was less than 3-fold, which is approximately half that measured in mice on regular diet (Figure 2C). Next, we measured HA levels in 4-MU-fed mice 14 days 
A
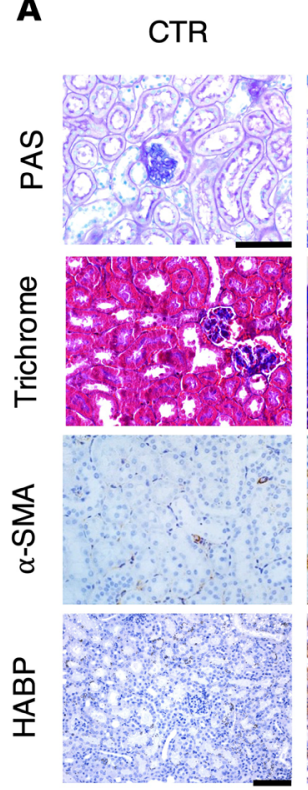
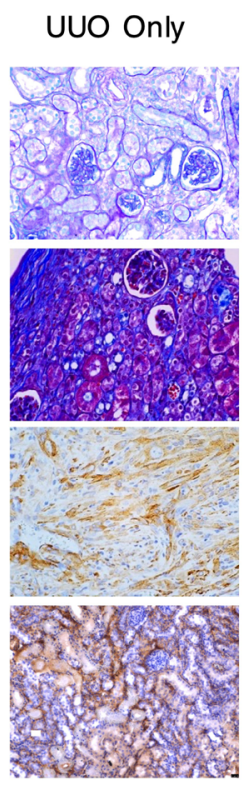

IL-10 Treated UUO
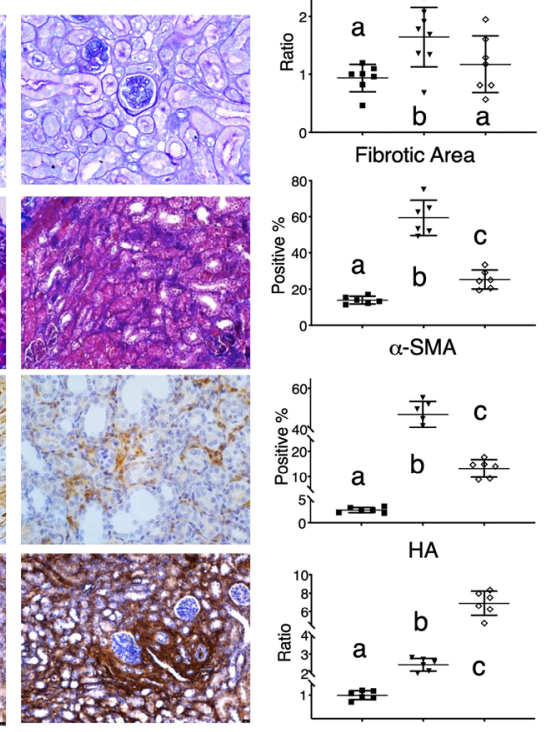

Has3
B Intratubular Space
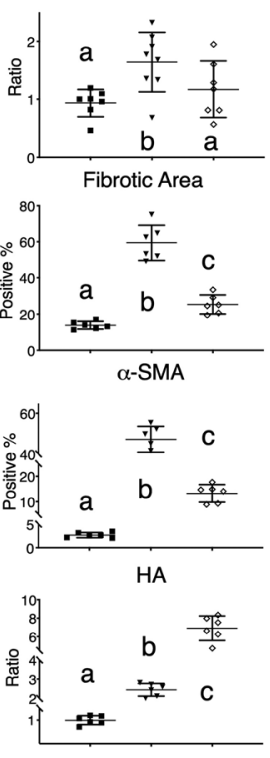

Has3

Hayl2
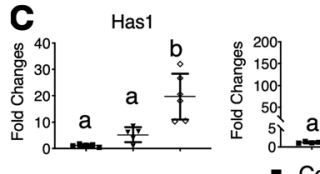

Has2

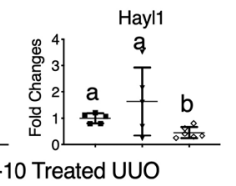

造
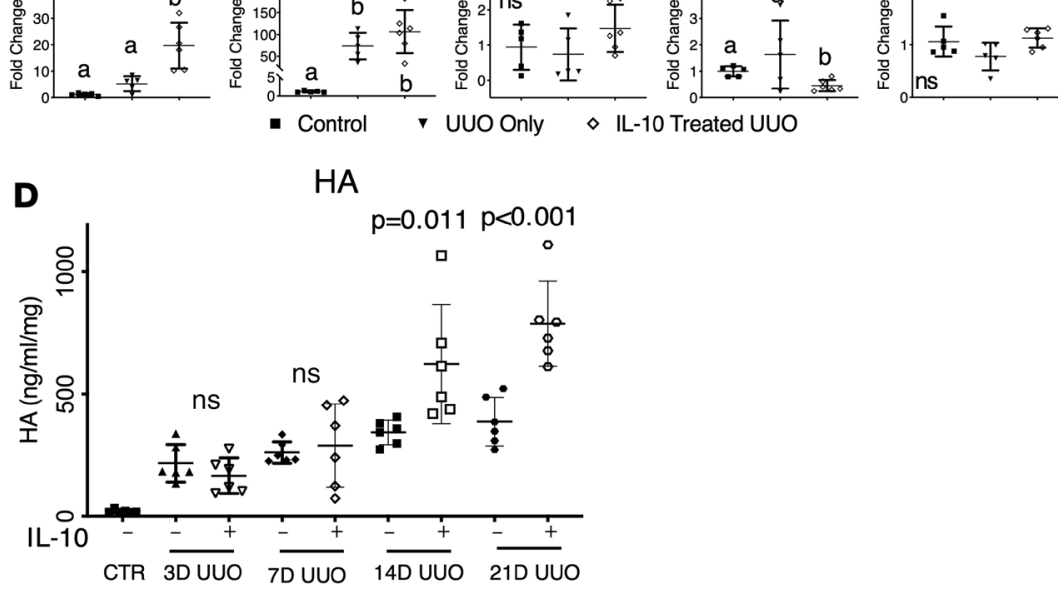

Figure 2. IL-10 upregulates HA synthesis in vivo, increases HMW-HA, and reduces renal fibrosis. (A) Images of PAS, trichrome, $\alpha$-SMA, and HABP staining in untreated controls, day 14 UUO, and IL-10-treated day 14 UUO kidneys. Scale bars: $75 \mu \mathrm{m}$ for PAS, trichrome, and $\alpha$-SMA staining; $100 \mu \mathrm{m}$ for HABP staining. (B) Quantification of respective PAS intratubular space, trichrome fibrotic area, $\alpha$-SMA, and HABP-stained HA levels from panel A. Data were analyzed by 1 -way ANOVA with post hoc Tukey's tests. Significantly different groups are not connected by the same letter. $P<0.01$ for fibrotic area and $\alpha$-SMA; $P<0.05$ for intratubular space and HA. (C) Relative mRNA level of Has1-3 and Hyal1-2 with control, day 14 UUO, and day 14 IL-10-treated UUO kidneys. $n \geq 3$ per sex per condition; $P<0.01$ for Has1 and Has2; $P<$ 0.05 for Hyal1. (D) HA tissue concentration in IL-10-treated UUO kidneys was sustained for at least 21 days. Tissue HA concentration $(\mathrm{ng} / \mathrm{mL} / \mathrm{mg})$ in untreated CTR and UUO kidneys with or without IL-10 treatment over time was measured by an ELISA-like HA assay. 21D UUO vs. IL-10-treated UUO ( $P<0.001)$; 14D UUO vs. IL-10-treated UUO ( $P=0.011)$; 3D and 7D of UUO vs. IL-10-treated UUO was not significant (ns). Data in $\mathbf{B}$ and $\mathbf{C}$ were analyzed by 1-way ANOVA with post hoc Tukey's test. Data in D were used for covariance (ANCOVA) analyses with the time points as a continuous covariate, while post hoc Tukey's tests were performed between the pairs at each time point. Groups annotated with different letters indicate they are significantly different. $P<0.01$ unless otherwise noted.

post-UUO, and, consistent with histology data, the data did not show significant differences in tissue HA levels irrespective of IL-10 treatment (Figure 4E). Moreover, 4-MU-fed UUO mice had lower total HA levels compared with their counterparts on the regular diet (Figure 1C). Taken together, these data further support our preceding conclusion that inhibition of endogenous HA synthesis by 4-MU selectively interrupts HA-dependent IL-10 antifibrotic effects.

IL-10-driven HMW-HA synthesis regulates kidney cell proliferation to attenuate fibrosis in $I / R$ mice. To determine whether IL-10 treatment could attenuate fibrosis in other CKD models, we investigated its effects in 


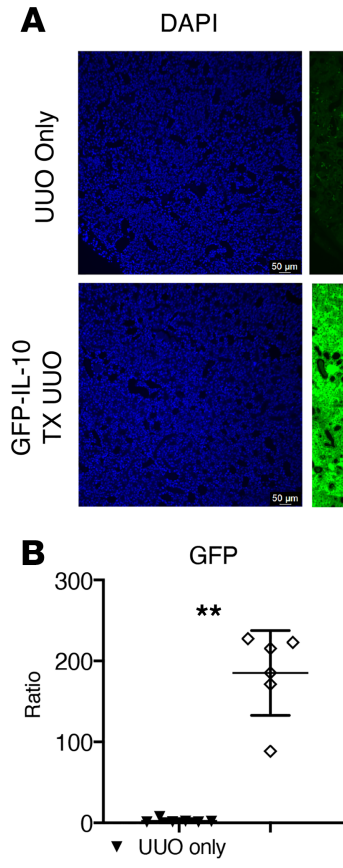

$\diamond$ GFP-IL-10 TX UUO
GFP
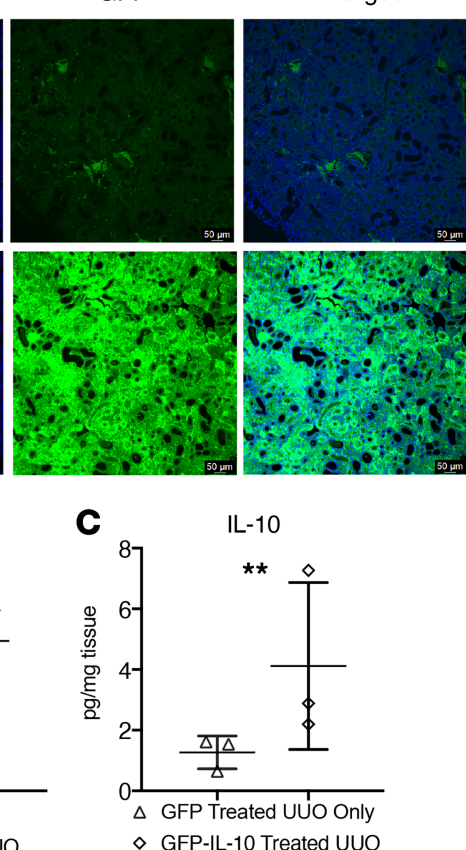

Figure 3. Increased IL-10 levels in kidney tissues are driven by lenti-GFP-IL-10 transduction. (A) Images of the kidney cortex at day 3 after UUO. Almost no GFP is observed in tissues transduced with control (empty GFP vector) versus strong signal from tissues treated with lenti-GFP-IL-10. Scale bars: $50 \mu \mathrm{m}$. (B) Quantification of imaging data was performed by measuring GFP intensity corresponding to immunofluorescence staining. ${ }^{* *} P<0.001$. (C) Tissue IL-10 concentration (pg/mg tissue weight) from day 3 lenti-GFP (control) UUO or day 3 lenti-GFP-IL-10-treated UUO kidneys was measured by an IL-10 ELISA test kit. ${ }^{* *} P<0.001$.

unilateral I/R. Images of trichrome and $\alpha$-SMA staining showed that IL-10-treated I/R kidneys exhibited reduced ECM deposition and $\alpha-\mathrm{SMA}^{+}$myofibroblasts compared with I/R-only kidneys (Figure 5A). Also, IL-10-treated I/R kidneys showed increased HA levels, which could be due to both I/R injury and IL-10 stimulation. Quantified fibrotic areas, $\alpha-\mathrm{SMA}^{+}$staining, and HA levels further supported our hypothesis that IL-10 overexpression can attenuate renal fibrosis in CKD models other than UUO (Figure 5B). Importantly, quantitative analysis of HA by an ELISA-like assay further revealed that in comparison with I/R-only, IL-10-treated kidneys had increased HA at matched time points (Figure 5C), where the combination of treatment and time was statistically significant $(P<0.01)$. Coherently, I/R-only kidneys had increased levels of HA on days 7 and 14, which decreased at day 30 after I/R. Thus, our results revealed that IL-10 treatment increased HA levels at 3 and 7 days after I/R, which may contribute to the reduced fibrotic and $\alpha-\mathrm{SMA}^{+}$area observed at 7 days with IL-10 treatment (Figure 5A).

We then determined the effect of IL-10 treatment on Has1-3 and hyaluronidase 1-2 mRNA levels at day 3 post I/R. All HA synthesis and degradation genes were upregulated in both untreated and IL-10treated I/R kidneys, although IL-10 treatment did result in increased Has3 versus I/R-only (Figure 5D). Interestingly, all Has and hyaluronidase mRNA levels in both groups were dramatically reduced at later time points (data not shown), indicating that the HA production driven by injury and IL-10 in the I/R model could be transient. We also noted that hyaluronidase 1 and 2 changes in the I/R model were larger than those of the UUO model, indicating higher HA turnover in I/R. Finally, we examined BrdU levels at day 3 in I/R kidneys with and without IL-10 treatment, which showed increased BrdU expression in the medulla versus the cortex (Figure 5, E and 5). Interestingly, IL-10-treated I/R kidneys showed more BrdU expression, especially in the interstitium, compared with I/R-only kidneys (Figure 5E). These results suggest that IL-10-treated I/R kidneys exhibited increased HA and reduced fibrosis, which could be due to IL-10-mediated cell proliferation in the interstitial area.

HA signaling in renal fibroblasts is partially regulated by IL-10/STAT3 signaling. In most organs, fibroblasts are the primary producers of ECM components, including HA. Since HA is produced at the cell membrane, it is considered a major component of the pericellular matrix. Thus, we investigated whether 
A

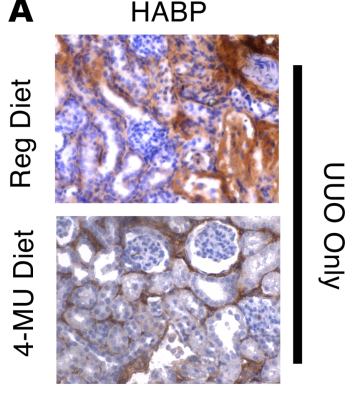

B

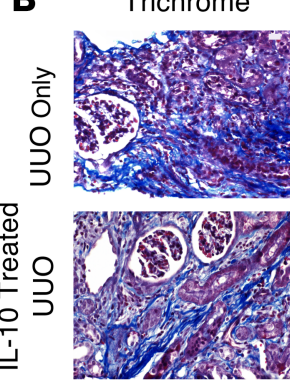

$\alpha$-SMA

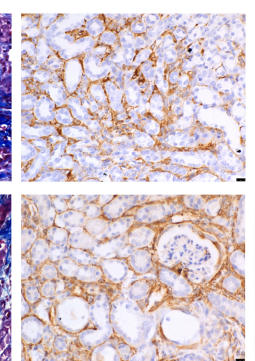

$\mathrm{F} 4 / 80$

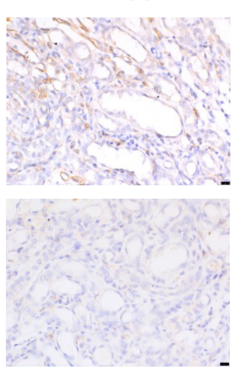

HABP

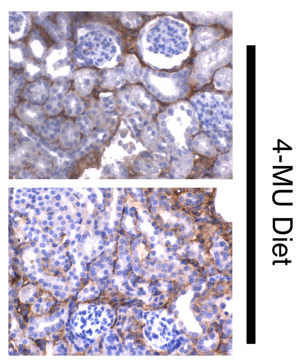

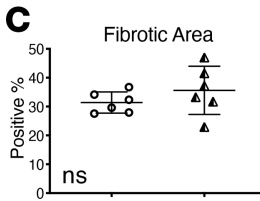
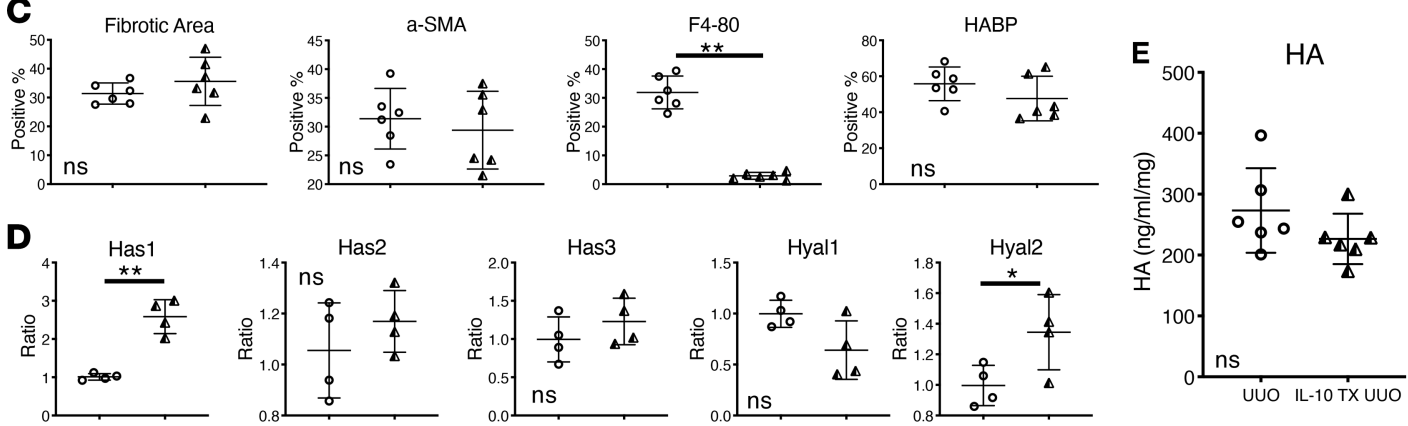

$\triangle 4-M U$ diet IL-10 TX UUO

- 4-MU diet UUO only

Figure 4. IL-10 antifibrotic effects depend on HA, while its antiinflammatory effects are independent. (A) HABP-stained kidney tissue images of day 14 post-UUO mice fed with either regular diet or 4-MU-supplemented diet. Scale bar: $50 \mu \mathrm{m}$. (B) Images of kidney cortex of mice fed the 4-MU diet, which underwent UUO in the absence or presence of IL-10. Trichrome, $\alpha$-SMA, and HA staining shows that treatment with IL-10 in the 4-MU diet group did not change the fibrotic phenotype, in contrast to previous results in the regular diet group. However, reduction of F4/80 (a marker of macrophage infiltration) suggested that inhibition of HA synthesis does not affect IL-10 antiinflammatory function. (C) Quantification of data from images of panel B as a percentage or ratio of each stain; ${ }^{* *} P<0.01$. (D) Relative mRNA levels of hyaluronan synthases (Has1-3) and hyaluronidases (Hyal1-2) of UUO and IL-10-treated UUO kidneys from 4-MU-fed mice; $n \geq 3$ per sex per condition; ${ }^{*} P<0.01$ for Has1; and ${ }^{*} P<0.05$ for Hyal2. (E) Tissue HA concentration (ng/mL/mg) as measured by an ELISA-like assay in UUO kidneys or IL-10-treated UUO kidneys from mice on 4-MU diet showed no significant difference. All data were analyzed with $t$ test for differences between groups; ns = no significant differences among groups.

renal fibroblasts produce HA in response to IL-10 treatment by performing particle exclusion assay to measure in vitro pericellular matrix formation. Figure 6A images show that IL-10-stimulated renal fibroblasts produced significantly larger pericellular matrix than controls (fibroblasts vs. IL-10-stimulated fibroblasts, $1.45 \pm 0.07$ vs. $2.70 \pm 0.11, P<0.05$ ). Then, when IL-10-stimulated renal fibroblasts were treated with hyaluronidase, we observed that pericellular matrix was reduced to control levels, indicating that HA is the primary component (Figure 6B).

Since STAT3 is recognized as a downstream signal transducer of IL-10/IL-10 receptor interactions to promote HA synthesis, we investigated how the mechanism operates in renal fibroblasts. We found that in comparison with control, fibroblasts treated with $100 \mathrm{ng} / \mathrm{mL}$ IL-10 exhibited 2.49-fold higher phosphorylated STAT3 (p-STAT3) and a 1.88-fold increase in Has2 (Figure 6, C and D), which mainly synthesizes HMW-HA $(P<0.05)$. Of note, $200 \mathrm{ng} / \mathrm{mL}$ IL-10 inhibited Has2 expression and STAT3 phosphorylation without changing Has1 levels at either dose, consistent with the published range for IL-10 pleiotropic effects (10-100 ng/mL) (50).

To identify the cell lineages that are prominently involved in the in vivo responses to renal injury, we performed IHC analyses using antibodies to identify kidney interstitial fibroblasts (FSP-1) and kidney proximal tubular cells (E-cadherin), with a costain for TIM-1, a marker of acute kidney injury. We found that lenti-IL-10-treated tissues at day 14 post-UUO expressed less TIM-1 than untreated UUO and that tubular cells expressed more TIM-1 than interstitial fibroblasts, indicating that tubular cells had a stronger injury response in the context of UUO (Figure 6E).

In sum, our results show that Has2 and p-STAT3 are upregulated by IL-10 in renal fibroblasts, indicating that HMW-HA production is induced by IL-10 via STAT3 signal transduction. We further identified that tubular cells exhibit a strong injury response in vivo, indicating that they may gain the most cytoprotective benefit from the increased production of antifibrotic HMW-HA in response to renal injury. 

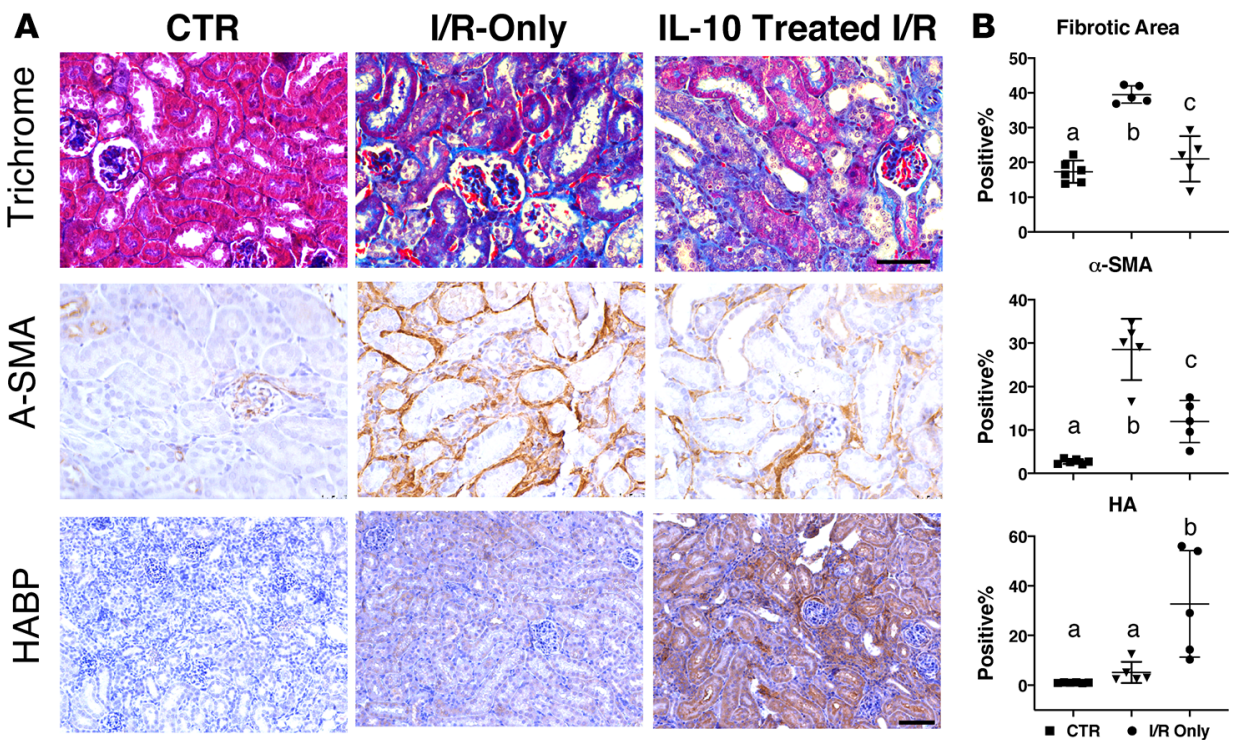

C

HA
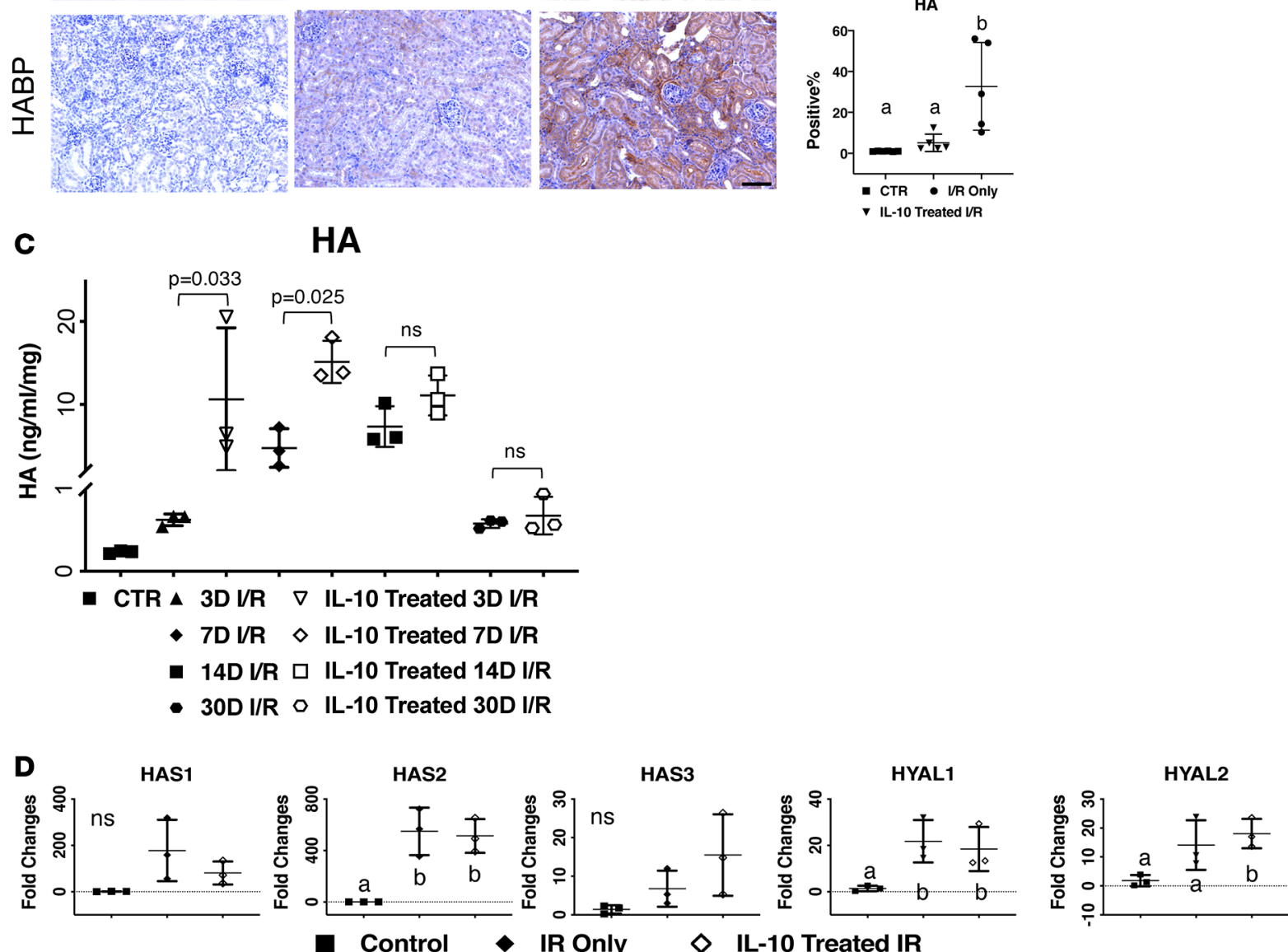

E
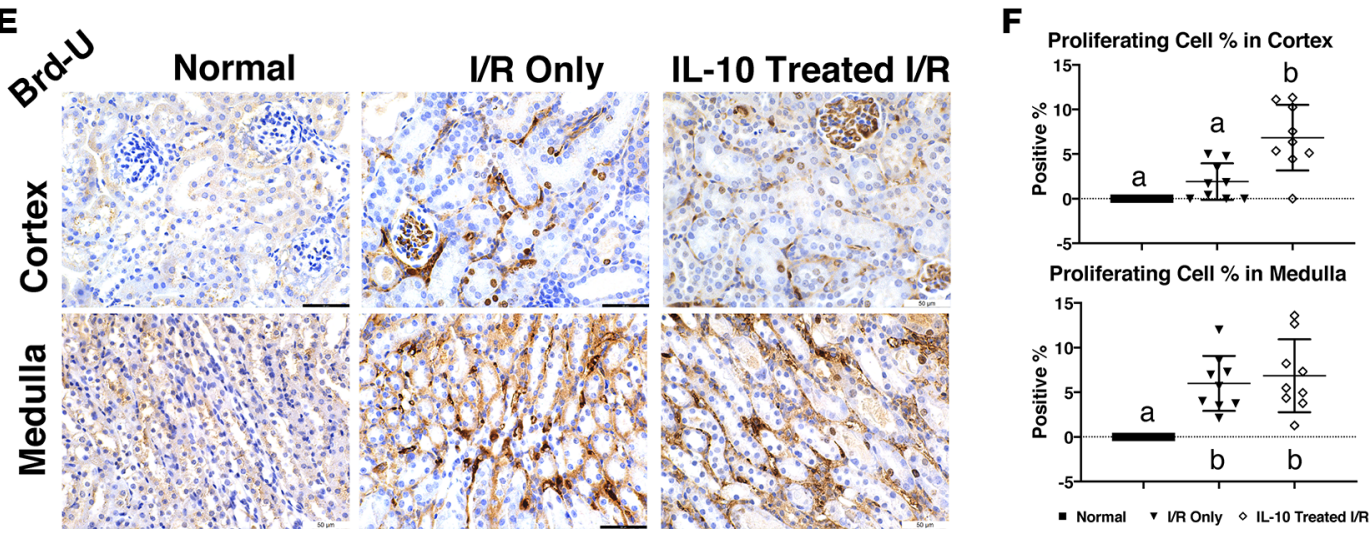
Figure 5. IL-10-driven HMW-HA synthesis and regulation of kidney cell proliferation attenuate fibrosis in I/R mice. (A) Images of trichrome and $\alpha$-SMA staining of control and day 7 I/R-only and IL-10-treated I/R tissue, with HABP staining of day 14 I/R-only and IL-10-treated I/R tissue. Scale bars: $75 \mu \mathrm{m}$ for trichrome and $\alpha$-SMA staining, $100 \mu \mathrm{m}$ for HABP staining. (B) Quantification of fibrotic area (trichrome), $\alpha$-SMA, and HABP from images of panel A. Data were analyzed with an unpaired $t$ test. (C) HA concentration $(\mathrm{ng} / \mathrm{mL} / \mathrm{mg})$ extracted from control and day 3,7 , and 14 post-UUO kidneys was measured by an HA test kit (modified ELISA). CTR vs. 3D/14D/30D ( $P<0.01)$; CTR vs. 7D $(P<0.05)$; 3D and 7D I/R vs. IL-10-treated I/R $(P<0.01) ; 14 D$ and 30D I/R vs. IL-10-treated I/R were not significant (ns). (D) Relative mRNA expression of Has1-3 and Hyal1-2 in normal kidney, I/R kidney, and IL-10treated I/R kidney. $n \geq 3$ per sex per condition, $P<0.05$. (E) Images of BrdU incorporation at day 3 post-I/R and IL-10-treated I/R in the kidney cortex and medulla. Scale bar: $50 \mu \mathrm{m}$. (F) Quantification of proliferating cells (\% positive cells) from cortex and medulla sections in normal, I/R-only, and IL-10treated I/R kidneys. $P<0.01$. Data from panels B, D, and $\mathbf{F}$ were analyzed by 1-way ANOVA with post hoc Tukey's test. Data from $\mathbf{C}$ were analyzed by covariance (ANCOVA) with the time points as a continuous covariate, and post hoc Tukey's tests were performed between the pairs at each time point. Groups annotated with different letters indicate significant differences at indicated $P$ values.

\section{Discussion}

To our knowledge, the present study provides the first evidence of IL-10-driven HMW-HA production in response to obstructive and ischemic renal injuries, the source of which is likely fibroblasts. We also noted that exogenous IL-10 further increased and upheld HMW-HA levels via upregulation of Has1 and 2 expression for at least 14 days post-UUO injury, which was accompanied by attenuated tubulointerstitial fibrosis. Extending these conclusions to other injury models, our I/R model confirmed that high HA levels are produced in response to injury and that IL-10 can increase HA production to reduce fibrosis. Figure 7 illustrates the pathway we are proposing based on our findings in the UUO model. Overall, our data suggest that HMW-HA synthesis plays a transient but protective physiologic role in the response to obstructive and ischemic injury, and this response can be reinforced and extended by IL-10 treatment. Leveraging the physiologic roles of HMW-HA and IL-10 could provide clues to design new antifibrotic therapies to resolve a major gap in the standard of care for patients with CKD.

We reported that IL-10 regulates and sustains HMW-HA levels to promote regenerative dermal tissue repair (11), which is supported by the evidence that loss of IL-10 leads to increased fibrosis in mouse models (51). IL-10 has been studied in other models of organ fibrosis, including lung and kidney (51-53), and is known to improve renal function and reduce inflammation after acute kidney injury $(43,54)$. This led us to investigate whether IL-10 could upregulate HMW-HA in the kidney to attenuate fibrosis, which could uncover a new antifibrotic role for IL-10 in CKD (51). In agreement with this reasoning, our present studies show that IL-10-mediated HA upregulation can reduce renal fibrosis via Has 2 and STAT3 signaling in renal fibroblasts. Our findings are further supported by earlier observations of increased HA levels in response to kidney injuries in other animal models of clinical disease (16). Moreover, the increase of HA in UUO is commensurate with the relative mRNA levels of all 3 Has enzymes, particularly Has1 and 2, which produce antiinflammatory HMW-HA variants. Liang et al. recently reported that knockdown of Has2 impairs lung renewal capacity in mice (55), and Has 2 has been shown to regulate TGF- $\beta$, a proinflammatory factor and key fibrosis mediator in several renal cell injury studies $(33,37,47)$.

Critically, our studies aimed to understand the balance between HA synthesis and degradation during the injury processes, as the balance between these processes determines HA MW, which in turn determines its physiologic function (pro- vs. antifibrotic). Our quantitative assessment of HA MW variants produced in the context of UUO showed that HMW-HA production is part of the initial physiologic response to kidney injury and provides evidence that these HMW-HA variants start to shift to lower MW variants after 7 days. This is supported by the increased expression of both synthesis and degradation enzymes in UUO. Declèves et al. reached similar conclusions in a study of unilateral renal I/R injury in rats; the authors attributed the elevated levels of HA in kidney to a combination of increased HMW-HA production postinjury and increased degradation (56). Later studies by the same team reported that HA inhibition by a 4-MU diet was protective against a renal I/R injury $(34,57)$; however, the authors did not appreciate the differential impact of distinct HA MW variants (20). Their approach to prevent HA synthesis is likely effective because it prevents the creation of profibrotic LMW-HA variants due to the action of hyaluronidases on the synthesized HA. By contrast, we show that IL-10 treatment can increase HMW-HA synthesis while preventing increased hyaluronidase expression. This shifts the balance of HA toward synthesis, upholding an antifibrotic, cytoprotective effect at longer time points. Indeed, we found upregulation of hyaluronidase genes in the I/R model in parallel with increased HA synthesis, which increased the LMW-HA variants that lead to long-term fibrotic outcomes $(P<0.05)$. 


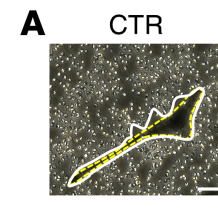

C

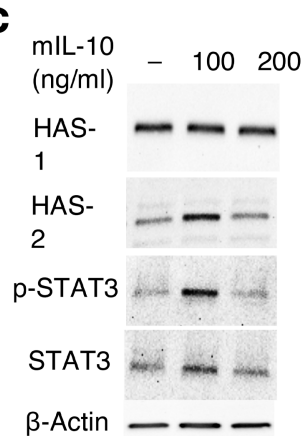

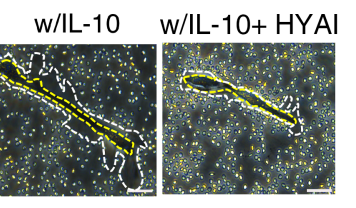
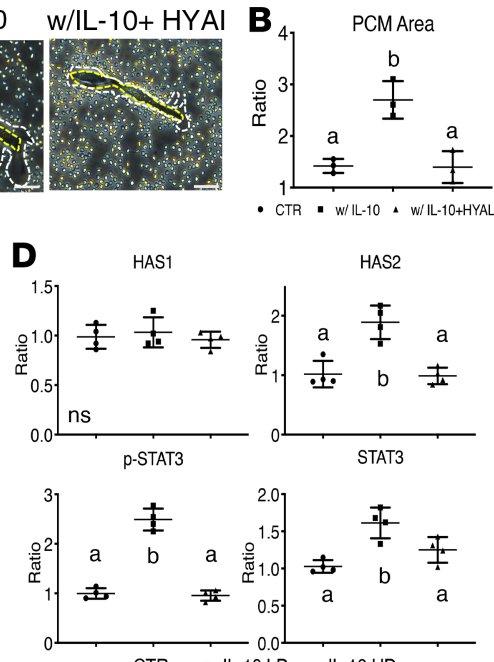

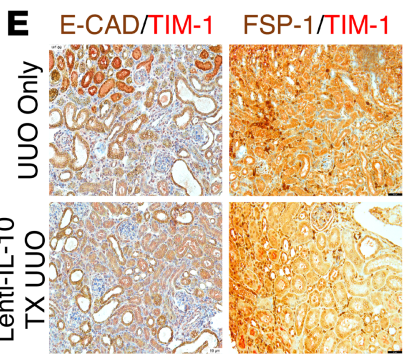

Figure 6. HA-driven attenuation of renal fibrosis is mediated by Has2- and STAT3-dependent mechanisms in renal fibroblasts. (A) Images of pericellular matrix (PCM) measurements on renal fibroblasts after a 24-hour incubation with media, IL-10 treatment, or IL-10 and hyaluronidase treatment. The yellow dashed line traces the cell body while the white dashed line traces the PCM area, as bordered by sheep red blood cells. (B) Quantification of renal fibroblast PCM area based on panel A images. (C) Immunoblotting of renal fibroblast Has1, Has2, p-STAT3, STAT3, and control $\beta$-actin protein levels after incubation in the presence or absence of IL-10. $P<0.0001$ for $p$-STAT3; $P<0.01$ for STAT3. (D) Adjusted quantification of immunoblotting data from panel C. (E) Representative images of E-CAD and TIM-1 expression colabeled on the cortex of day 7 UUO or day 7 lenti-GFP-IL-10-treated UUO by IHC and representative images of FSP-1 and TIM-1 expression colabeled on the cortex of day 14 UUO or day 14 lenti-GFP-IL-10-treated UUO by IHC. Scale bars: $50 \mu \mathrm{m}$. All data were analyzed by 1-way ANOVA with post hoc Tukey's test for differences among groups. Groups not connected by the same letter are statistically different at the indicated $P$ value.

In both of our models, we observed that IL-10 promoted HMW-HA synthesis and stability (58), leading to our working hypothesis that IL-10 induces and upholds renal HMW-HA production in a measured and transient fashion to promote regenerative wound healing.

Our present study focuses on the potential pathophysiology of the renal fibroblast responses to injury; however, we did note that Has1 and Has2 levels can be different in vivo (Figure 1D) and in vitro (Figure 4D). Therefore, we could not rule out the potential involvement of other cell lineages or critical pathways in tubulointerstitial fibrosis. For instance, Kang et al. reported that renal tubular epithelial cells contribute to the development of fibrotic tubules in a large cohort study (59). Future studies could address the potential role of other kidney cells in HA-mediated mechanisms of renal fibrosis, interrogate STAT3 signaling by suppressing STAT3 and/or Has2, and generate kidney cell-specific Has2-knockout mouse models. Such studies would further allow us to uncover how HA MW variants differentially regulate the direction of renal responses to injury toward regenerative tissue repair or fibrosis.

In the interim, we sought to determine whether our studies on UUO are relevant to other CKD models. We examined a unilateral I/R model with 40 minutes of ischemia to mimic acute kidney injury that can lead to regenerative wound healing or fibrosis. Indeed, the reduced fibrosis and increased HA levels in IL-10-treated I/R kidneys support the conclusions drawn from the UUO model. We are aware that the inherent nature of the unilateral I/R model limits HA accumulation, which, while measurable via IHC, is not sufficient to assess MW distribution by size-exclusion chromatography or gel electrophoresis. 


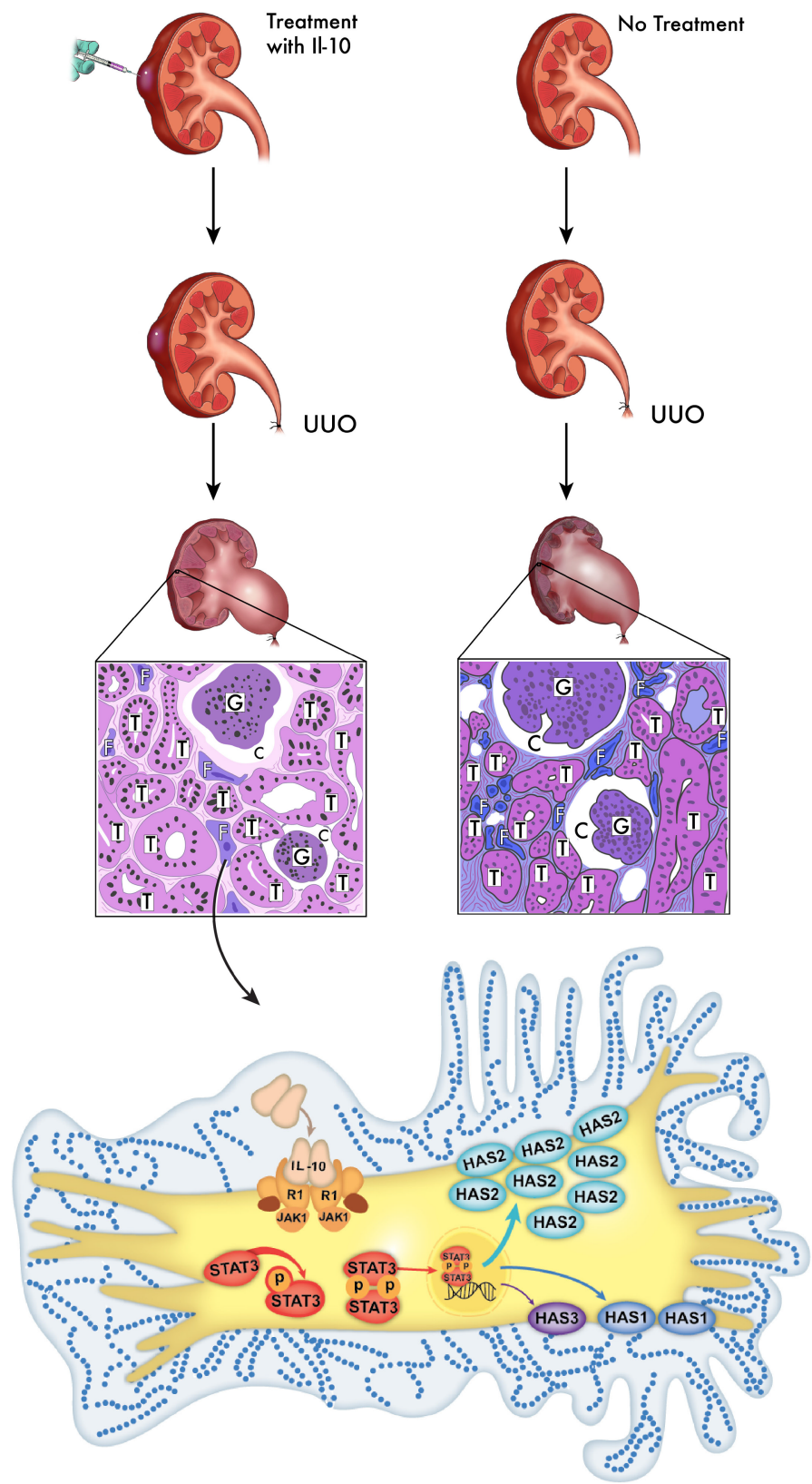

Figure 7. Schematic illustration of renal tubulointerstitial fibrosis attenuated by HMW-HA. As illustrated here, we performed UUO procedures to model injury in mouse kidneys and harvested the tissues after 14 days. Kidneys treated with IL-10 demonstrated reduced fibrosis and better preserved tubules, whereas untreated UUO kidneys showed more activated profibrotic renal myofibroblasts. This figure illustrates how IL-10 stimulates renal fibroblasts, activates the STAT3 pathway, and upregulates HA synthesis (predominantly HMW via Has2) to ultimately result in cytoprotection and less fibrosis. T, tubule; $\mathrm{G}$, glomeruli; C, capsular space; F, fibroblast. The schematic is a broad illustration that is not drawn to scale.

Other I/R models, such as bilateral I/R (60) or nephrectomy (61), may need to be specifically redesigned and applied to produce more extreme fibrotic responses that facilitate quantification of HA MW changes. Consistent with studies by Chao et al. (62), we observed an impact of injury on epithelial cell proliferation in the interstitial area at the medullar part of the I/R kidney (Figure 5). Indeed, others have reported similar findings, namely that HA can promote proliferation in pulmonary tissues and wounds $(63,64)$. Undoubtedly, more work is needed to determine the pro- or antifibrotic characteristics of these proliferating interstitial cells in the I/R model and explain their overall significance in fibrotic outcomes (65). Our data suggest that HA synthesis and IL-10 share common mechanisms of injury response, which may be implicated in pathologies that end in CKD and renal fibrosis. 
In sum, our present study underscores 3 fundamental physiologic factors: (a) HA MW matters (20); (b) cytokines and HMW-HA function in concert to promote and sustain antiinflammatory, antifibrotic, and cytoprotective effects; and (c) UUO and I/R highlight bona fide pathophysiologic models to assess IL-10/ HMW-HA concerted responses to renal injury. We conclude that regulation of Has and hyaluronidase results in preferential synthesis, and stability of HMW-HA has transient but sufficient cytoprotective effects in response to renal fibrosis. Further studies on renal fibroblasts and the potential role of STAT3 in human and animal models of CKD will contribute a better understanding of the pathophysiology of fibrosis, in addition to how crosstalk between interstitial fibroblasts and tubular cells could influence the outcome of wound healing. These results provide a proof of concept to support the bench-to-bedside work of HMWHA and IL-10 regulatory mechanisms into adjuvant therapies for CKD.

\section{Methods}

Animal studies. C57BL/6J mice (The Jackson Laboratory 000664) were purchased, bred, and maintained in the Texas Children's Hospital Feigin Center vivarium under pathogen-free conditions with access to food and water ad libitum.

UUO and treatment. We adapted a well-established UUO model of renal fibrosis (66). Briefly, 8- to 10-week-old male and female C57BL/6J mice fed with normal or 4-MU diet underwent UUO procedures in identical anatomic locations on their bilateral flanks, wherein 1 ureter was doubly ligated with sutures at the proximal end. The contralateral kidney remained untreated as an internal control. During surgery, mice were sedated with 2\% isoflurane (Henry Schein Animal Health NDC 11695-6776-2) inhaled intranasally. Mouse muscle and skin layers were sutured separately with 4-0 vicryl and 6-0 prolene sutures (Ethicon J386h and 8711h), respectively. Kidney samples were harvested 3, 7, 14, or 21 days later. Buprenorphine (BSRLAB1 ZooPharm) was administered for pain management.

For lentivirus transduction, $1 \times 10^{9}$ transducing units of lenti-IL-10-GFP or lenti-GFP genetically engineered lentivirus (CMV promoter) constructs (53) were diluted up to $20 \mu \mathrm{L}$ with pharmaceutical-grade saline and injected under the kidney capsule of mouse cohorts with a 29-gauge insulin syringe (BD 329424J) (11). UUO procedures were performed 3 or 4 days after lenti-IL-10-GFP injection to ensure relevant expression of the cytokine. Wound dressings (Tegaderm, $3 \mathrm{M}$ ) were applied to prevent postoperative complications. Concentrated lenti-IL-10-GFP and lenti-GFP were prepared by Baylor College of Medicine Vector Development Lab.

4-MU (MilliporeSigma M1381) was pressed into the mouse chow at 5\% (wt/wt) by TestDiet and irradiated before shipment, as previously described (33).

Renal unilateral $I / R$ injury and treatment. We induced renal unilateral I/R in 8- to 10-week-old male C57BL/6J mice according to a previously published protocol that we adapted for the present study (60). Briefly, the left-side renal artery and vein were isolated and occluded with microaneurysm clamp (15913, World Precision Instruments) for 40 minutes. During surgery, mice were sedated with $2 \%$ isoflurane inhaled intranasally. After removal of the clamp, mouse muscle and skin layers were sutured separately with 4-0 vicryl and 6-0 prolene, respectively. Kidney samples were harvested 3, 7, or 14 days later. Buprenorphine was administered for pain management. We used the same method of lenti-IL-10-GFP construct injection to induce IL-10 expression in the I/R model as in the UUO model, and we performed I/R injury 3 or 4 days after lenti-IL-10-GFP injection. Wound dressings were applied to prevent postoperative complications.

$H A$ extraction for gel electrophoresis and ELISA. To extract HA from our renal samples, we adapted a protocol based on previous literature, including a protocol developed by Cleveland Clinic with support from NIH P01HL107147 (67-69). Briefly, mouse kidney tissues (medulla and cortex) were weighed and treated with $1 \mathrm{mg} / \mathrm{mL}$ proteinase K (Invitrogen, Thermo Fisher Scientific) at $25 \mathrm{mg}$ tissue $/ \mathrm{mL}$, then incubated at $60^{\circ} \mathrm{C}$ for 4 hours. Samples were vortexed every 30 minutes until tissues were completely dissociated. Four volumes of prechilled 200-proof ethanol were added to each sample, and samples were kept at $-20^{\circ} \mathrm{C}$ overnight. The second day, samples were centrifuged at $14,000 \mathrm{~g}$ for 10 minutes at room temperature, and the resulting pellets were washed with 4 volumes of prechilled $75 \%$ ethanol $(\mathrm{EtOH})$ before being vortexed. The centrifugation process was repeated, and then pellets were allowed to air-dry at room temperature for 20 minutes. Then, $100 \mathrm{mM}$ ammonium acetate was added to each sample, which was then vortexed and spun down. Proteinase $\mathrm{K}$ was inactivated by heat at $100^{\circ} \mathrm{C}$ for 5 minutes, and samples were chilled on ice for 5 minutes. Samples were incubated at $37^{\circ} \mathrm{C}$ overnight with $1 \mu \mathrm{L}$ of Benzonase (MilliporeSigma), after which the enzyme was inactivated at $95^{\circ} \mathrm{C}$ for 5 minutes. Four volumes of cold 200-proof EtOH 
were added to each sample and left to precipitate overnight at $-20^{\circ} \mathrm{C}$. On the fourth day, samples were centrifuged at $14,000 \mathrm{~g}$ for 10 minutes at room temperature. The pellet was washed with $1 \mathrm{~mL}$ of cold $75 \%$ ethanol and centrifuged again. Finally, samples were air-dried and resuspended in $20 \mu \mathrm{L} 100 \mathrm{mM}$ ammonium acetate ( $\mathrm{pH} 7.0$ ) and loaded onto $0.5 \%$ agarose gel. All reagents were purchased from Thermo Fisher Scientific unless otherwise noted.

Primary cell isolation and culture. Primary C57BL/6J murine kidney fibroblasts were purchased from CellBiologics (N.C57-6016). Passage 4-10 fibroblast cultures were maintained at $37^{\circ} \mathrm{C}$ under $5 \% \mathrm{CO}_{2}$ in a humidified chamber with DMEM/F12 supplemented with $10 \%$ fetal bovine serum (Corning) and $1 \times$ penicillin-streptomycin.

For all experimental procedures, cells were subcultured and settled overnight in growth medium. Cells were washed with $1 \times$ PBS twice before being treated with serum-free culture medium for 16 hours. Various treatments were then added to the conditioned medium ( $n \geq 3$ per time point). All reagents are from Thermo Fisher Scientific unless otherwise noted.

Particle exclusion assay and morphometric analysis of pericellular matrix. Pericellular matrix analysis was performed as described previously (11). Briefly, fibroblasts were plated at a density of $1.5 \times 10^{5}$ cells/well in 6-well plates (BD Falcon) and then left untreated or exposed to murine IL-10 (100 ng/mL or $200 \mathrm{ng} / \mathrm{mL}$; PeproTech) for 24 hours. To determine whether the pericellular matrix contained HA, $1 \mathrm{U} / \mathrm{mL}$ of hyaluronidase from Streptomyces hyalurolyticus (H1136; MilliporeSigma) was added to the culture medium for 20 minutes after the initial 24-hour incubation. Cells were washed in PBS, and $500 \mu \mathrm{L}$ of glutaraldehyde-stabilized sheep erythrocytes $\left(1 \times 10^{8}\right.$ cells $/ \mathrm{mL}$; Intercell Technologies $)$ was added to each well to visualize fibroblast pericellular matrix. The cell body and pericellular matrix were outlined as a halo around the cells bounded by red blood cells. Pericellular matrix ratio was calculated as the ratio of total pericellular matrix area to the cell body area (20-30 cells analyzed per condition).

Real-time quantitative PCR. Quantitative PCR was performed as previously described (11). Briefly, tissues were lysed to isolate total RNA with RNeasy Micro Kit (QIAGEN). cDNA was synthesized using a High-Capacity Reverse Transcription Kit (Applied Biosystems, Thermo Fisher Scientific). Gene expression was assayed in triplicate using Power SYBR Green PCR Master Mix with a StepOnePlus Real-Time PCR System (Applied Biosystems, Thermo Fisher Scientific). Gene expression was normalized against mouse GAPDH. Relative expression values were calculated using the $\Delta \triangle \mathrm{C}_{\mathrm{T}}$ method. Oligonucleotide primer sequences are listed in Supplemental Table 1; see also Supplemental Data and Supplemental Figures 1 and 2; supplemental material available online with this article; https://doi. org/10.1172/jci.insight.136345DS1. Mouse Has2 and hyaluronidase 1 primers (Bio-Rad 10025636, qMmuCID0011459, and qMmuCED0040215) were purchased from Bio-Rad Laboratory.

HA extraction and Hyaluronic Acid Test Kit (an ELISA-like assay). Mouse kidney sections, including medulla and cortex, were weighed and digested in freshly made $\mathrm{pH}$-optimized lysis buffer containing 0.15 M Tris base, 0.15 M NaCl, 0.01 $\mathrm{M} \mathrm{CaCl}_{2}, 5 \mathrm{mM}$ deferoxamine mesylate, and $20 \mathrm{U} / \mathrm{mL}$ Streptomyces griseus protease (MilliporeSigma) in a ratio of $15 \mu \mathrm{L}$ buffer per $1 \mathrm{mg}$ of tissue (70). Samples were digested at $55^{\circ} \mathrm{C}$ for 16 hours while shaking at $1400 \mathrm{rpm}$. Protease was denatured at $95^{\circ} \mathrm{C}$ for 15 minutes to stop digestion and the sample supernatant was collected. HA quantification was performed using a Hyaluronic Acid Test Kit (Corgenix).

ELISA. Kidney tissues were weighed and lysed in $150 \mu \mathrm{L}$ of Pierce RIPA buffer (Thermo Fisher Scientific 89900) containing 1\% protease inhibitor (MilliporeSigma P8340) and 1\% phosphatase inhibitors (MilliporeSigma P5726). Tissue lysates were subjected to a mouse IL-10 quantikine ELISA kit (R\&D Systems, Bio-Techne, M1000B).

Size-exclusion chromatography and hyaluronic acid ELISA-like assay. Analysis of HA MW was performed as described previously (71). Briefly, kidney tissues were lyophilized overnight, weighed, and digested with Proteinase $\mathrm{K}(250 \mu \mathrm{g} / \mathrm{mL})$ in $100 \mathrm{mM}$ ammonium acetate $\mathrm{pH} 7.0$ at $60^{\circ} \mathrm{C}$ overnight. After digestion, the enzyme was inactivated by heating to $95^{\circ} \mathrm{C}$ for 15 minutes. Total concentration of HA per tissue weight was determined by a modified competitive HA ELISA, where the samples were first mixed with biotinylated HABP (MilliporeSigma 385911) and then added to microtiter plates (BD Falcon) coated with bovine HA (gift from Wight's laboratory, Seattle, Washington, USA). The final signal is inversely proportional to the concentration of HA in the sample. An analytical Sephacryl S-1000 column (GE Healthcare Life Sciences) was used to assess the size of HA (46, 72). A 200- $\mu$ L aliquot containing 6-7 $\mu$ g of isolated HA was applied to an analytical Sephacryl S-1000 column. HA was eluted 
from the column with $1 \times \mathrm{PBS}$ at a flow rate of $12-15 \mathrm{~mL} / \mathrm{h}$ and $0.3-\mathrm{mL}$ fractions were collected. Upon completion, fractions were quantitated by the HA ELISA-like assay to generate a profile.

Immunoblotting. C57BL/6J murine kidney fibroblasts were lysed in RIPA buffer (Thermo Fisher Scientific), and digested samples were run on a $4 \%-12 \%$ Tris-glycine gel (Mini-PROTEAN TGX Stain-Free Precast Gels) and transferred to a nitrocellulose membrane using a Trans-Blot Turbo Transfer System. All protein electrophoresis and blotting experiments were performed using Bio-Rad Laboratory products. Membranes were blocked with $5 \%$ bovine serum albumin in $1 \times$ Tween TBS and incubated with primary antibodies antiHAS1 (Abcam 198846), anti-HAS2 mAb (Abcam 199794), rabbit anti-STAT3 (79D7), or rabbit anti-p-STAT3 (Tyr705) mAb (D3A7) (Cell Signaling Technology 9145) at $4^{\circ} \mathrm{C}$ overnight, or with $\beta$-actin (Cell Signaling Technology 4970) at room temperature for 1 hour, then incubated with HRP-linked secondary antibodies (Abcam ab97080 and Cell Signaling Technology 7076) at room temperature for 1 hour. All primary antibodies were diluted 1:1000. Protein bands were visualized with ECL (Pierce, Thermo Fisher Scientific) and imaged by BioRad ChemiDoc XRS+ (Bio-Rad). Band densitometry was performed using Image Lab image analysis software using $\beta$-actin as a loading control. See full-length immunoblotting images in Supplemental Figure 3.

Histology and IHC. Tissues were dehydrated, paraffin-embedded, and cut into 5- $\mu$ m-thick sections. PAS and trichrome kits were purchased from Leica Biosystems and used with a Leica ST5020 Autostainer. A standard IHC protocol was followed for all stains, which were performed using a Dako Autostainer Link 48 (Agilent) and its accompanying software (Dakolink version 4.1, edition 3.1.0.987, Agilent). Primary antibodies against $\alpha$-SMA (Abcam 5694), F4/80 (Abcam 111101), and BrdU (MilliporeSigma, B2531) were detected by EnVision+System-HRP (DAB) kit (Agilent). HABP staining, adapted from a previous publication (35), was performed using HABP (MilliporeSigma, 385911), streptavidin (MilliporeSigma, S-5512), and ImmPACT DAB Peroxidase (HRP) Substrate kit (Vector Labs, SK-4105) to detect HA. For double staining, TIM-1 polyclonal antibody (Invitrogen, Thermo Fisher Scientific, PA5-98302) was detected by EnVision+System-HRP (AEC) kit (Agilent), and E-cadherin (Cell Signaling Technology 3195) and FSP-1 (MilliporeSigma 07-2274) were detected by EnVision+System-HRP (DAB) kit (73).

Immunofluorescence staining. We adapted the immunofluorescence staining protocol from the study by Cheng et al. (74). Briefly, tissues were dehydrated, embedded, and cut into 5- $\mu \mathrm{m}$-thick samples that were mounted onto slides. Sections were deparaffinized and blocked with 5\% goat serum in PBS for 1 hour at room temperature. Primary antibodies were diluted in PBS with 1\% bovine serum albumin. For GFP staining, we used anti-GFP antibody (Abcam 183734) and goat anti-rabbit IgG (H+L) cross-adsorbed secondary antibody, cyanine3 (Invitrogen, Thermo Fisher Scientific A10520).

For GFP expression, we followed the protocol by Nakagawa et al. (75). Briefly, paraffin slides were gently agitated in 2 changes of prechilled xylenes and 2 changes of prechilled $95 \% \mathrm{EtOH}$, followed by 3 changes of prechilled TBS and final mounting with DAPI medium.

Microscopy. Histology slides were imaged with a Leica DM2000 microscope using Leica application Suite $\mathrm{X}$ version 3.0.4.16529. Immunofluorescence slides were imaged with a Leica DMi8 fluorescent microscope using Leica application Suite X version 3.0.4.16319.

Statistics. Statistical analysis was performed with GraphPad Prism 8.2.1 using 1-way ANOVA and/ or multiple linear regression (ANCOVA, for data in Figure 2D and Figure 4C) when the number of groups exceeded 2 or 2-tailed mean $t$ test when numbers of groups equaled 2 . All quantitative data are expressed as mean $\pm \mathrm{SD}$, and $P \leq 0.05$ was considered statistically significant.

Study approval. The animal protocol was approved by the Institutional Animal Care and Use Committee at Baylor College of Medicine.

\section{Author contributions}

XW and SGK designed the study; XW, EHS, SRM, AJB, HL, CKC, TCL, and MMR performed experiments; XW, SB, SGK, EHS, CKC, HL, PLB, and JC analyzed data; XW made the figures; XW, EHS, AJB, and SGK drafted/revised the paper; SGK, PLB, JC, TNW, SB, SRM, and PFA provided scientific comments for the paper; and all authors discussed the results and commented on the manuscript.

\section{Acknowledgments}

This work was supported by Texas Children's Hospital Department of Surgery Clayton Award (TCPSO WANG X 2018 SURG SEED) to XW, NIH R01GM111808, and a gift from Amie and Jeff Springmeyer to support research in regenerative medicine to SGK. 
We thank the Office of Surgical Research Administration at Texas Children's Hospital (Hector Martinez-Valdez and Monica Fahrenholtz) for editorial support; Yu Ling, Aditya Kaul, Chad Moles, Mathangi A. Chandramouli, Yao Ning, Meghana Kodali (Houston Bellaire High School), and Leighanne Masri (University of California, Santa Barbara) for technical assistance; Qi Wei within the Office of Surgical Research of the Baylor College of Medicine Department of Surgery for statistical support; Jill Tuley for administrative assistance; Virginia M. Green for editorial assistance; and Shelia Hall, David Aten (MD Anderson Cancer Center), and Rebecca Shon Bower for their work on the illustrations.

Address correspondence to: Sundeep G. Keswani, Texas Children's Hospital and Baylor College of Medicine, Feigin Center, C450.06, 1102 Bates Avenue, Houston, Texas 77030, USA. Phone: 832.822.3135; Email: keswani@bcm.edu.

1. Cox TR, Erler JT. Remodeling and homeostasis of the extracellular matrix: implications for fibrotic diseases and cancer. Dis Model Mech. 2011;4(2):165-178.

2. Nanthakumar CB, Hatley RJ, Lemma S, Gauldie J, Marshall RP, Macdonald SJ. Dissecting fibrosis: therapeutic insights from the small-molecule toolbox. Nat Rev Drug Discov. 2015;14(10):693-720.

3. Liu Y. Renal fibrosis: new insights into the pathogenesis and therapeutics. Kidney Int. 2006;69(2):213-217.

4. Liu Y. Cellular and molecular mechanisms of renal fibrosis. Nat Rev Nephrol. 2011;7(12):684-696.

5. Zhou D, Liu Y. Renal fibrosis in 2015: Understanding the mechanisms of kidney fibrosis. Nat Rev Nephrol. 2016;12(2):68-70.

6. Kopp JB, et al. MYH9 is a major-effect risk gene for focal segmental glomerulosclerosis. Nat Genet. 2008;40(10):1175-1184.

7. Manson SR, Niederhoff RA, Hruska KA, Austin PF. Endogenous BMP-7 is a critical molecular determinant of the reversibility of obstruction-induced renal injuries. Am J Physiol Renal Physiol. 2011;301(6):F1293-F1302.

8. Manson SR, Niederhoff RA, Hruska KA, Austin PF. The BMP-7-Smad1/5/8 pathway promotes kidney repair after obstruction induced renal injury. J Urol. 2011;185(suppl 6):2523-2530.

9. Singh S, et al. Tubular Overexpression of Angiopoietin-1 Attenuates Renal Fibrosis. PLoS One. 2016;11(7):e0158908.

10. Balaji S, et al. The role of interleukin-10 and hyaluronan in murine fetal fibroblast function in vitro: implications for recapitulating fetal regenerative wound healing. PLoS One. 2015;10(5): 0124302.

11. Balaji S, et al. Interleukin-10-mediated regenerative postnatal tissue repair is dependent on regulation of hyaluronan metabolism via fibroblast-specific STAT3 signaling. FASEB J. 2017;31(3):868-881.

12. Shamskhou EA, et al. Hydrogel-based delivery of Il-10 improves treatment of bleomycin-induced lung fibrosis in mice. Biomaterials. 2019;203:52-62.

13. Jung M, et al. IL-10 improves cardiac remodeling after myocardial infarction by stimulating M2 macrophage polarization and fibroblast activation. Basic Res Cardiol. 2017;112(3):33.

14. Zeisberg M, Neilson EG. Mechanisms of tubulointerstitial fibrosis. J Am Soc Nephrol. 2010;21(11):1819-1834.

15. Levey AS, Coresh J. Chronic kidney disease. Lancet. 2012;379(9811):165-180.

16. Albeiroti S, Soroosh A, de la Motte CA. Hyaluronan's role in fibrosis: a pathogenic factor or a passive player? Biomed Res Int. 2015;2015:790203.

17. Tampe B, et al. Low-dose hydralazine prevents fibrosis in a murine model of acute kidney injury-to-chronic kidney disease progression. Kidney Int. 2017;91(1):157-176.

18. Yang C, et al. The high and low molecular weight forms of hyaluronan have distinct effects on CD44 clustering. J Biol Chem. 2012;287(51):43094-43107.

19. Sapudom J, et al. Molecular weight specific impact of soluble and immobilized hyaluronan on CD44 expressing melanoma cells in 3D collagen matrices. Acta Biomater. 2017;50:259-270.

20. Cyphert JM, Trempus CS, Garantziotis S. Size matters: molecular weight specificity of hyaluronan effects in cell biology. Int J Cell Biol. 2015;2015:563818.

21. Bollyky PL, Bogdani M, Bollyky JB, Hull RL, Wight TN. The role of hyaluronan and the extracellular matrix in islet inflammation and immune regulation. Curr Diab Rep. 2012;12(5):471-480.

22. Itano $\mathrm{N}$, et al. Three isoforms of mammalian hyaluronan synthases have distinct enzymatic properties. J Biol Chem. 1999;274(35):25085-25092.

23. Tian X, et al. High-molecular-mass hyaluronan mediates the cancer resistance of the naked mole rat. Nature. 2013;499(7458):346-349.

24. Noble PW. Hyaluronan and its catabolic products in tissue injury and repair. Matrix Biol. 2002;21(1):25-29.

25. Stern R, Jedrzejas MJ. Hyaluronidases: their genomics, structures, and mechanisms of action. Chem Rev. 2006;106(3):818-839.

26. Csoka AB, Frost GI, Stern R. The six hyaluronidase-like genes in the human and mouse genomes. Matrix Biol. 2001;20(8):499-508

27. Hansell P, Göransson V, Odlind C, Gerdin B, Hällgren R. Hyaluronan content in the kidney in different states of body hydration. Kidney Int. 2000;58(5):2061-2068.

28. Göransson V, Johnsson C, Jacobson A, Heldin P, Hällgren R, Hansell P. Renal hyaluronan accumulation and hyaluronan synthase expression after ischaemia-reperfusion injury in the rat. Nephrol Dial Transplant. 2004;19(4):823-830.

29. Rügheimer L, Olerud J, Johnsson C, Takahashi T, Shimizu K, Hansell P. Hyaluronan synthases and hyaluronidases in the kidney during changes in hydration status. Matrix Biol. 2009;28(7):390-395.

30. Colombaro V, et al. Hyaluronidase 1 and hyaluronidase 2 are required for renal hyaluronan turnover. Acta Histochem. 2015;117(1):83-91.

31. Johnsson C, Hällgren R, Wahlberg J, Tufveson G. Renal accumulation and distribution of hyaluronan after ureteral obstruction. 
Scand J Urol Nephrol. 1997;31(4):327-331.

32. Pijuan-Galitó S, et al. Human serum-derived protein removes the need for coating in defined human pluripotent stem cell culture. Nat Commun. 2016;7:12170.

33. Kuipers HF, et al. The pharmacokinetics and dosing of oral 4-methylumbelliferone for inhibition of hyaluronan synthesis in mice. Clin Exp Immunol. 2016;185(3):372-381.

34. Kultti A, et al. 4-Methylumbelliferone inhibits hyaluronan synthesis by depletion of cellular UDP-glucuronic acid and downregulation of hyaluronan synthase 2 and 3. Exp Cell Res. 2009;315(11):1914-1923.

35. Nagy $\mathrm{N}$, et al. Inhibition of hyaluronan synthesis restores immune tolerance during autoimmune insulitis. J Clin Invest. 2015;125(10):3928-3940.

36. Hascall VC, et al. The dynamic metabolism of hyaluronan regulates the cytosolic concentration of UDP-GlcNAc. Matrix Biol. 2014;35:14-17.

37. Nagy N, et al. 4-methylumbelliferone treatment and hyaluronan inhibition as a therapeutic strategy in inflammation, autoimmunity, and cancer. Front Immunol. 2015;6:123.

38. Strutz F, Zeisberg M. Renal fibroblasts and myofibroblasts in chronic kidney disease. J Am Soc Nephrol. 2006;17(11):2992-2998.

39. Lin SL, Kisseleva T, Brenner DA, Duffield JS. Pericytes and perivascular fibroblasts are the primary source of collagen-producing cells in obstructive fibrosis of the kidney. Am J Pathol. 2008;173(6):1617-1627.

40. Henderson NC, et al. Galectin-3 expression and secretion links macrophages to the promotion of renal fibrosis. Am J Pathol. 2008;172(2):288-298

41. Liu Y. New insights into epithelial-mesenchymal transition in kidney fibrosis. J Am Soc Nephrol. 2010;21(2):212-222.

42. Rodell CB, Rai R, Faubel S, Burdick JA, Soranno DE. Local immunotherapy via delivery of interleukin-10 and transforming growth factor $\beta$ antagonist for treatment of chronic kidney disease. J Control Release. 2015;206:131-139.

43. Soranno DE, et al. Delivery of interleukin-10 via injectable hydrogels improves renal outcomes and reduces systemic inflammation following ischemic acute kidney injury in mice. Am J Physiol Renal Physiol. 2016;311(2):F362-F372.

44. Djudjaj S, Boor P. Cellular and molecular mechanisms of kidney fibrosis. Mol Aspects Med. 2019;65:16-36

45. Heinegård D, Hascall VC. Aggregation of cartilage proteoglycans. 3. Characteristics of the proteins isolated from trypsin digests of aggregates. J Biol Chem. 1974;249(13):4250-4256.

46. Bogdani M, Simeonovic C, Nagy N, Johnson PY, Chan CK, Wight TN. The detection of glycosaminoglycans in pancreatic islets and lymphoid tissues. Methods Mol Biol. 2015;1229:413-430.

47. Nagy N, Kuipers HF, Marshall PL, Wang E, Kaber G, Bollyky PL. Hyaluronan in immune dysregulation and autoimmune diseases. Matrix Biol. 2019;78-79:292-313.

48. Dahl LB, Kimpton WG, Cahill RN, Brown TJ, Fraser RE. The origin and fate of hyaluronan in amniotic fluid. J Dev Physiol. 1989;12(4):209-218.

49. Roughley PJ, White RJ, Glant TT. The structure and abundance of cartilage proteoglycans during early development of the human fetus. Pediatr Res. 1987;22(4):409-413.

50. Schmit A, et al. Dose-effect of interleukin-10 and its immunoregulatory role in Crohn's disease. Eur Cytokine Netw. 2002;13(3):298-305.

51. $\mathrm{Mu} \mathrm{W}$, et al. IL-10 suppresses chemokines, inflammation, and fibrosis in a model of chronic renal disease. J Am Soc Nephrol. 2005;16(12):3651-3660.

52. Kurosaki F, et al. AAV6-mediated IL-10 expression in the lung ameliorates bleomycin-induced pulmonary fibrosis in mice. Hum Gene Ther. 2018;29(11):1242-1251

53. Peranteau WH, et al. IL-10 overexpression decreases inflammatory mediators and promotes regenerative healing in an adult model of scar formation. J Invest Dermatol. 2008;128(7):1852-1860.

54. Jin Y, Liu R, Xie J, Xiong H, He JC, Chen N. Interleukin-10 deficiency aggravates kidney inflammation and fibrosis in the unilateral ureteral obstruction mouse model. Lab Invest. 2013;93(7):801-811.

55. Liang J, et al. Hyaluronan and TLR4 promote surfactant-protein-C-positive alveolar progenitor cell renewal and prevent severe pulmonary fibrosis in mice. Nat Med. 2016;22(11):1285-1293.

56. Declèves AE, et al. Synthesis and fragmentation of hyaluronan in renal ischaemia. Nephrol Dial Transplant. 2012;27(10):3771-3781.

57. Colombaro V, et al. Inhibition of hyaluronan is protective against renal ischaemia-reperfusion injury. Nephrol Dial Transplant. 2013;28(10):2484-2493.

58. Conti P, et al. IL-10, an inflammatory/inhibitory cytokine, but not always. Immunol Lett. 2003;86(2):123-129.

59. Kang HM, et al. Defective fatty acid oxidation in renal tubular epithelial cells has a key role in kidney fibrosis development. Nat Med. 2015;21(1):37-46.

60. Kim Y, et al. Elevated urinary CRELD2 is associated with endoplasmic reticulum stress-mediated kidney disease. JCI Insight. 2017;2(23):92896

61. Li Z, et al. (Pro)renin receptor is an amplifier of Wnt/B-catenin signaling in kidney injury and fibrosis. J Am Soc Nephrol. 2017;28(8):2393-2408.

62. Chao H, Spicer AP. Natural antisense mRNAs to hyaluronan synthase 2 inhibit hyaluronan biosynthesis and cell proliferation. J Biol Chem. 2005;280(30):27513-27522.

63. Li Y, et al. Hyaluronan synthase 2 regulates fibroblast senescence in pulmonary fibrosis. Matrix Biol. 2016;55:35-48.

64. Meran S, et al. Hyaluronan facilitates transforming growth factor-beta1-mediated fibroblast proliferation. J Biol Chem. 2008;283(10):6530-6545.

65. Wynn TA, Ramalingam TR. Mechanisms of fibrosis: therapeutic translation for fibrotic disease. Nat Med. 2012;18(7):1028-1040.

66. Liang M, et al. Protective role of insulin-like growth factor-1 receptor in endothelial cells against unilateral ureteral obstruction-induced renal fibrosis. Am J Pathol. 2015;185(5):1234-1250.

67. Bhilocha S, et al. Agarose and polyacrylamide gel electrophoresis methods for molecular mass analysis of 5- to 500-kDa hyaluronan. Anal Biochem. 2011;417(1):41-49.

68. Lee HG, Cowman MK. An agarose gel electrophoretic method for analysis of hyaluronan molecular weight distribution. Anal Biochem. 1994;219(2):278-287. 
69. van Gemst JJ, et al. RNA contaminates glycosaminoglycans extracted from cells and tissues. PLoS One. 2016;11(11):e0167336.

70. Armstrong SE, Bell DR. Measurement of high-molecular-weight hyaluronan in solid tissue using agarose gel electrophoresis. Anal Biochem. 2002;308(2):255-264.

71. Wilkinson TS, Potter-Perigo S, Tsoi C, Altman LC, Wight TN. Pro- and anti-inflammatory factors cooperate to control hyaluronan synthesis in lung fibroblasts. Am J Respir Cell Mol Biol. 2004;31(1):92-99.

72. Reed MJ, Damodarasamy M, Chan CK, Johnson MN, Wight TN, Vernon RB. Cleavage of hyaluronan is impaired in aged dermal wounds. Matrix Biol. 2013;32(1):45-51.

73. Terada N, Karim MR, Izawa T, Kuwamura M, Yamate J. Immunolocalization of $\beta$-catenin, E-cadherin and N-cadherin in neonate and adult rat kidney. J Vet Med Sci. 2017;79(11):1785-1790.

74. Cheng CF, et al. A fragment of secreted Hsp90 $\alpha$ carries properties that enable it to accelerate effectively both acute and diabetic wound healing in mice. J Clin Invest. 2011;121(11):4348-4361.

75. Nakagawa A, Alt KV, Lillemoe KD, Castillo CF, Warshaw AL, Liss AS. A method for fixing and paraffin embedding tissue to retain the natural fluorescence of reporter proteins. BioTechniques. 2015;59(3):153-155 